\title{
Single crystal growth of organic photoconductors: phthalocyanine
}

\author{
FRANCIS P XAVIER* and GEORGE J GOLDSMITH \\ Physics Department, Boston College, Chestnut Hill, MA 02167, USA \\ * Present address: Physics Department, Loyola College, Madras 600034, India \\ MS received 27 September 1995; revised 15 February 1996
}

\begin{abstract}
An effective method of growing single crystals of organic photoconductors such as phthalocyanine in the presence of doping impurity such as iodine by vacuum sublimation is discussed in this paper. This method is very useful especially when an organic material does not have a melting point but decomposes above a particular temperature. So far, doping has been done by exposing the previously grown organic single crystals to the dopant vapour, but growing the crystal in the presence of dopant vapour makes the doped-crystal more efficient and stable. The photosensitivity as well as the photoconductivity of the doped crystals by this method increases significantly.
\end{abstract}

Keywords. Crystal growth; doping organic semiconductor; photoconductor.

\section{Introduction}

Some aromatic hydrocarbons, such as anthracene and phthalocyanine $(\mathrm{Pc})$, were found to possess semiconducting properties (Inokuchi 1989). Their conductivity and mobility, however, when compared with those of inorganic semiconductors, are very low. A comparison of organic semiconductor $(\mathrm{Pc})$ properties with those of the elementary semiconductor (germanium) is given in table 1. Other compounds such as polyacetylene exhibit metallic conductivity and some organic superconductors have been reported (Hsu and Labes 1974; Baughman et al 1977; Gutmann and Lyons 1981).

The Pcs represent large conjugate systems, i.e. alternating double and single bonds (Orti et al 1960), which enhance photoconductivity. The molecule is planar (Orti and Bredas 1988; Schoch et al 1988) consisting of four isoindole molecules linked together at the corners of the pyrrole rings by four nitrogen atoms (figure 1). The space within the four central nitrogen atoms may be occupied either by hydrogen (metal free, HPc) or by any of the several metallic atoms (metal substituted, MPcs).

The Pcs exist in at least three different (polymorphic) modifications, the most common of which are designated alpha and beta (differing slightly both in crystal structure and electrical properties) (Gutmann and Lyons 1981; Schoch et al 1988). The alpha form occurs as polycrystalline films when sublimed under vacuum onto cool (room temperature) substrate. The beta form is crystallized into single crystals by vacuum sublimation at a temperature around $460^{\circ} \mathrm{C}$. Pcs crystallize in the form of long base centred monoclinic needles (Lyons et al 1960) with the long axis corresponding to the crystallographic b-axis.

Pcs have similar central structure as other semiconducting materials such as porphyrin, chlorophyll, and hemoglobin. The structural similarity of Pcs to chlorophyll, their stability against thermal and chemical decomposition, and their intense optical absorption in the visible spectrum (especially in the blue-green region) have resulted in their investigations for artificial solar energy cells (Orti et al 1990) and in textile and paint industries as dyes (Orti et al 1988). Primary cells using charge transfer 
Table 1. Comparison of organic semiconductor properties with those of an inorganic semiconductor (Heilmeier and Warfield 1963; Moser and Thomas 1963; Gamo et al 1968; Streetman 1980).

\begin{tabular}{lcc}
\hline & $\begin{array}{c}\text { Inorganic } \\
\text { semiconductors } \\
\text { (germanium) }\end{array}$ & $\begin{array}{c}\text { Organic } \\
\text { semiconductor } \\
\text { (phthalocyanine) }\end{array}$ \\
\hline Band gap (eV) & 0.67 & 1.6 \\
Mobility $\left(\mathrm{cm}^{2} /\right.$ V.sec) & 3900 & 1 \\
Carrier concentration $\left(\mathrm{cm}^{-3}\right)$ & $2 \cdot 5 \times 10^{13}$ & $10^{6}$ to $10^{7}$ \\
Trap density $\left(\mathrm{cm}^{-3}\right)$ & 43 & $10^{12}$ to $10^{14}$ \\
Resistivity (ohm.cm) & 16 & $10^{14}$ \\
Dielectric constant & 5.32 & 3.8 \\
Density (g/cm & & 1.6 \\
\hline
\end{tabular}

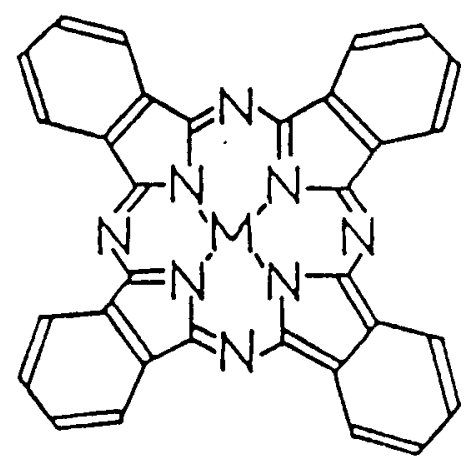

Figure 1. Structure of phthalocyanine.

complexes are now available as cardiac pacemakers (Lyons et al 1989). The Pcs are expected to serve as active materials for molecular electronics such as chemical sensor (Minami et al 1987).

\section{Experimental}

\subsection{Single crystal growth}

Pyrex tube $(20 \mathrm{~cm}$ long and $3.5 \mathrm{~cm}$ diam) having one end closed and the other end tapering $(10 \mathrm{~cm}$ long and $1 \mathrm{~cm}$ in diam) was cleaned well. The commercially available phthalocyanine (Aldrich - 98\%) powder (about $3 \mathrm{~g}$ ) was filled carefully so that no dry powder would stick to the inner wall of the pyrex tube where the crystals would start growing. The tapered part was then sealed to the vacuum system and the system pumped down to a pressure of the order of $10^{-5}$ torr. During evacuation the lower end of the pyrex tube where the sample powder was located was heated to about $340-385^{\circ} \mathrm{C}$ (above this temperature the sample decomposed) so that the unwanted impurities could be pumped away. Evacuation was continued for one week after which the sample 
tube was sealed off and removed from the vacuum system. The evacuated pyrex tube containing Pc in powder form was inserted into a temperature controlled oven (leaving $2 \mathrm{~cm}$ exposed to room temperature). The temperature near the sample was maintained constant $\left(460^{\circ} \mathrm{C}\right)$. After a day or so the crystals started growing at a suitable region in the temperature gradient about $15 \mathrm{~cm}$ from the bottom of the tube (Fielding 1957). After about 6 weeks, crystals were found in the form of clusters of hundreds of whiskers with dimensions $15 \sim 20 \times 0.3 \times 0.13 \mathrm{~mm}$. The length of a few crystals was longer $(3 \sim 4 \mathrm{~cm})$ than the reported dimension of a few mm (Aoyagi et al 1971; Cox et al 1974). The crystals were pinkish by reflected light and deep blue by transmitted light. The single crystallinity of the grown samples was confirmed.

\subsection{Iodine doped single crystal growth}

The doping has been so far done by first growing the single crystals by vacuum sublimation and then exposing them to the iodine vapour for a few hours (Grigoryan et al 1984; Waclawek et al 1987; Zabkowska-Waclawek et al 1987; Harikumar et al 1989). In the present study Pc crystals were grown in the presence of iodine vapour. For the purpose of introduction of iodine, the pyrex cylinder in which the crystals were to be grown was fitted with a side arm. Pc powder was placed in the cylinder as described above and a few crystals of iodine were placed in the side arm. The pyrex tube was connected to the vacuum system. Because of the high vapour pressure of iodine the vacuum pressure could not be brought below about 40 milli torr. With the use of a liquid nitrogen trap to lower the iodine vapour pressure, the pressure was brought to a few milli torr before sealing off. The main tube was inserted into the oven leaving the side arm (containing iodine) outside the oven near the room temperature. Crystal growth was then allowed to proceed. The temperature at the point of the Pc powder was kept at $460^{\circ} \mathrm{C}$. The colour of the crystal was dark red. The iodine doped crystal was tested for the presence of free iodine by rinsing some of them with tri-chloro ethylene. The characteristic purple colour of free iodine was not observed. Even when the crystal was heated up to $100^{\circ} \mathrm{C}$ the characteristics (conductivity) did not change. These observations indicate that the doped iodine atoms were permanently incorporated into the lattice of the Pc crystal.

Iodine was also introduced into single crystals of pure $\mathrm{H}_{2} \mathrm{Pc}$ by diffusion. In a crystallization tube similar to that used for growth of crystals in the presence of iodine, crystals of $\mathrm{H}_{2} \mathrm{Pc}$ were introduced into the main section and iodine crystals into the side arm. After evacuating and sealing, the tube was inserted into the temperature controlled oven as described above. The temperature at the sample was kept at $350^{\circ} \mathrm{C}$ for a few days. The crystals were then removed from the tube. While inside the evacuated crystallization tube the crystals appeared to have a silvery surface, but after a few hours in the atmosphere they regained their original pinkish colour.

\section{Results and discussion}

Pc crystallizes in the form of long base centred monoclinic prismatic (Lyons et al 1960) needles with the long axis corresponding to the crystallographic b-axis. All the samples investigated, both undoped and iodine doped, exhibited pronounced photoconductivity along the b-axis, while no photoconductive response was observed perpendicular 
to this direction. For this reason the Pc crystals could be called one-dimensional crystal (Wright 1989). Studies of time dependence, intensity dependence, electric field dependence, spectral dependence, and thermal behaviour of the photoconductivity were carried out.

Photoconductivity measurements were carried out with the sample (kept in vacuum) connected with an electrometer (Keithley 602) and with a potential of $500 \mathrm{~V}$ applied to the electrodes on the crystals separated by a gap of 1 to $2 \mathrm{~mm}$ along the b-axis. The light source was a 100-watt quartz-halogen lamp which was focussed through a monochromator onto the sample under study.

\subsection{Photocurrent vs wavelength}

The output of light from the monochromator between $350 \mathrm{~nm}$ and $1250 \mathrm{~nm}$ by means of stepping motor drive was incident on the sample and the corresponding photocurrent in the circuit was measured. The photoconductivity spectrum over the range between 1 and $3.5 \mathrm{eV}$ exhibits one large maximum at $1.6 \mathrm{eV}$ having a width at half maximum of $0.17 \mathrm{eV}$, and a broader large maximum at $2.5 \mathrm{eV}$ having about half the amplitude of the first peak with a half width of $0.41 \mathrm{eV}$. There are three additional smaller peaks at $1 \cdot 1,1 \cdot 2$, and $1 \cdot 8 \mathrm{eV}$. The photoconductivity maxima are ascribed to the band edges.

\subsection{Photocurrent vs time}

The measuring circuit consisted of the electrometer which had a response time $<1 \mathrm{~m} \mathrm{sec}$ which was sufficiently short to follow accurately the rise and decay of photocurrent. The output of the electrometer was led either to a strip chart recorder $\left(\tau_{\mathrm{r}}=0.1 \mathrm{sec}\right.$; maximum chart speed, $30 \mathrm{~cm} / \mathrm{min}$ ) or to a CRO fitted with Polaroid camera system. In most of the measurements the actual light source was a monochromator (Instruments SA) set at zero order fitted with the camera shutter. The measurements were carried out at room temperature. The sample was first illuminated and then the shutter was closed while recording the decay of the photocurrent. After the current reached its minimum value, the shutter was opened and the steady state current was allowed to rise till it reached a steady state once more. The rise and decay times were measured between the $10 \%$ and $90 \%$ points.

The time dependence of the photoconductivity at the onset and removal of excitation are exponential. The rising portion corresponds to a single exponential relation, between amplitude and time, of the form

$$
I_{\mathrm{t}}=I_{0} \exp \left\{-t / \tau_{\mathrm{r}}\right\}
$$

where $t$ is the time, $\tau_{r}$ the characteristic time constant. But the decay portion corresponds to a double exponential according to the relation

$$
I_{\mathrm{t}}=I_{0}\left\{\exp \left(-t / \tau_{1}\right)+\exp \left(-t / \tau_{2}\right)\right\}
$$

Simultaneous illumination of the crystal with infrared light (wavelength $>1100 \mathrm{~nm}$ ) resulted in an approximate doubling of the photocurrent, no change in the rise time but a $30 \%$ reduction in the decay time. 
Table 2. Comparison of iodine-doped crystal (HPcI) with undoped crystal $\left(\mathrm{H}_{2} \mathrm{Pc}\right)$.

\begin{tabular}{lll}
\hline & \multicolumn{1}{c}{$\mathrm{HPcI}$} & \multicolumn{1}{c}{$\mathrm{H}_{2} \mathrm{Pc}$} \\
\hline Dark current (amp) & $4.48 \times 10^{-12}$ & $3.15 \times 10^{-11}$ \\
Photocurrent (amp) & $1.86 \times 10^{-8}$ & $1.12 \times 10^{-9}$ \\
Rise time (sec) & 1.37 & 0.1 \\
Decay time (sec) & 2.20 & 0.09 \\
\hline
\end{tabular}
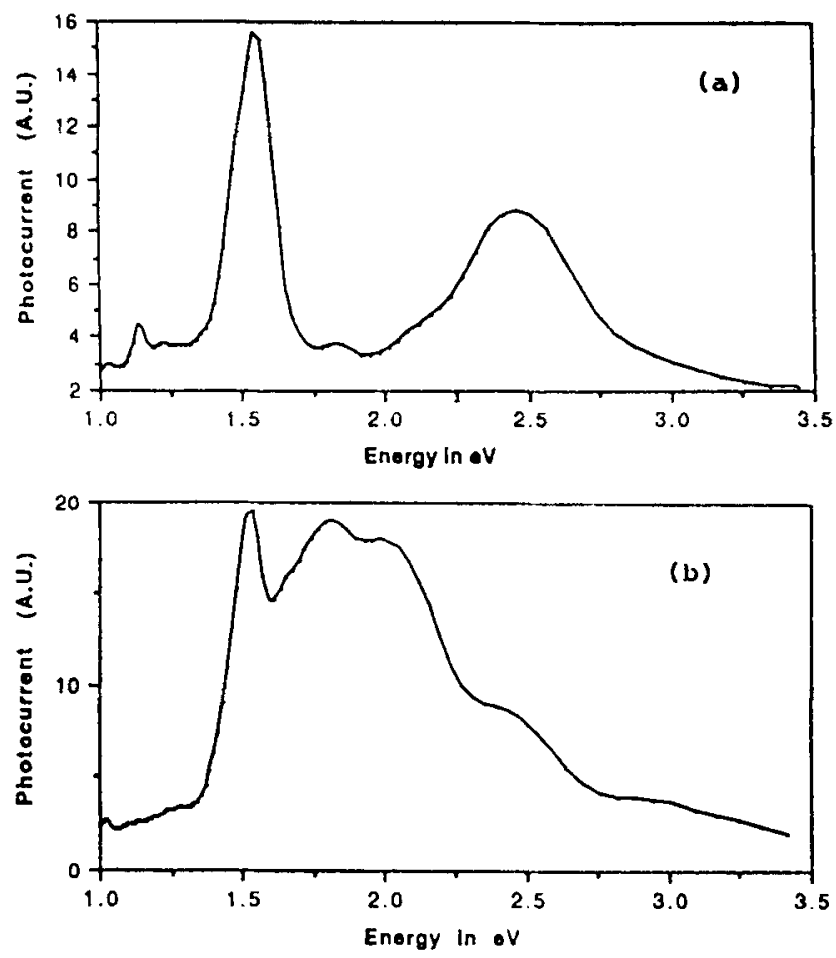

Figure 2. a. Spectral dependence of photoconductivity spectrum ef undoped single crystal of $\mathrm{H}_{2} \mathrm{Pc}$, and $\mathbf{b}$. spectral dependence of photoconductivity of iodine-doped single crystal of $\mathrm{HPcI}$.

\subsection{Photocurrent vs temperature}

For the study of the temperature dependence of photoconductivity, the sample was mounted on a small copper plate $(1 \mathrm{~cm} \times 2.5 \mathrm{~cm})$ that was uniformly coated with high thermal conductive epoxy adhesive (Omega Engineering) to provide uniform thermal conduction and electrical insulation. One end of the crystal of Pc (oriented along the b-axis) was fixed onto this surface using silver paint and a thin copper wire was attached to this crystal end to serve as an electrode. Since the thermal expansion of the crystal and that of the copper plate were different, the second electrode contact (spaced 1 to $2 \mathrm{~mm}$ ) was attached to the free end of the crystal. A heater wrapped around the thermal conductor and a constantin-Cu thermocouple at the crystal were provided to increase the temperature and to monitor the temperature at the crystal respectively. 


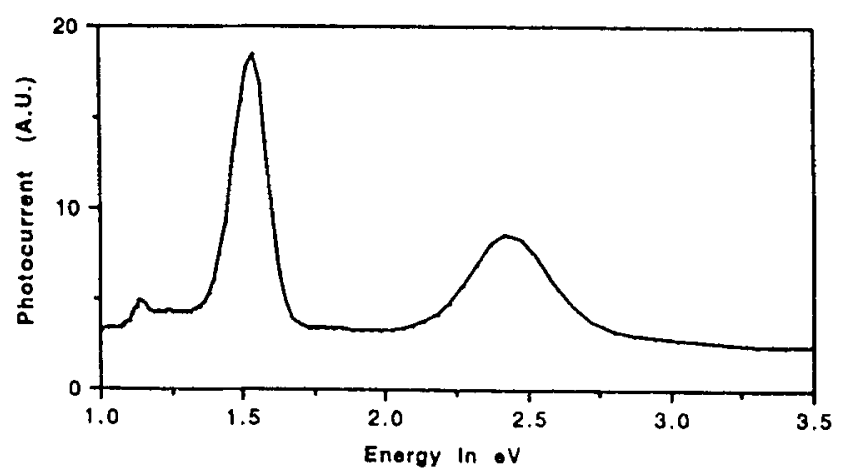

Figure 3. Spectral dependence of photocurrent of iodine-doped (by diffusion) singie crystal of $\mathrm{HPcI}(\mathrm{D})$.

Over the range, $100-300^{\circ} \mathrm{K}$, the photoconductivity, $\sigma$, follows the relation (Liang 1962)

$$
\sigma=\sigma_{0}\left\{\exp \left(-E_{\mathrm{t}} / k T\right)\right\}+C
$$

where $T$ is the temperature, $E_{\mathrm{t}}$ the activation energy, and $C$ represents dark current plus leakage current.

\subsection{Effect of iodine doping}

Upon the introduction of iodine into the crystals by growth in the presence of iodine vapour, the electrical properties are dramatically altered. For the iodine doped phthalocyanine crystal (HPcl) the dark conductivity decreases by about one order of magnitude while the photocurrent under the same illumination condition as the undoped $\left(\mathrm{H}_{2} \mathrm{Pc}\right)$ is greater by one order (table 2$)$. Spectral dependence of undoped single crystals of Pc (figure $2 a$ ) is compared with that of the doped crystals (figure $2 b$ ) (Xavier et al 1995). The barrier between $1.6 \mathrm{eV}$ and $2 \cdot 3 \mathrm{eV}$ of undoped crystal $\left(\mathrm{H}_{2} \mathrm{Pc}\right)$ is removed in the doped crystal $(\mathrm{HPcl})$ thereby enhancing the conductivity in the doped crystals. Even when the doped crystals were heated upto $100^{\circ} \mathrm{C}$ the dopant (iodine) did not escape thereby indicating that the dopant was embedded permanently into the crystal.

The photoconductivity spectrum of iodine-doped single crystal by diffusion $[\mathrm{HPcI}(\mathrm{D})]$ is shown in figure 3 which is similar to that of undoped crystal (figure 2a).

\section{Conclusion}

The method of growing organic photoconductive crystals by vacuum sublimation is very effective. This vacuum sublimation method in particular is very useful especially when the material, such as some of the organic photoconductors, does not have a melting point but decomposes above a particular temperature. Halogen doping (i.e. electron acceptors) makes the organic semiconductors (especially phthalocyanine which is $p$-type) more photosensitive. Thus, through suitable doping inter-molecular 
energy barrier in an organic photoconductor could be partially or fully eliminated and correspondingly photoconductivity could be enhanced. In the case of metal free Pc the energy barrier between $1.5 \mathrm{eV}$ and $2.5 \mathrm{eV}$ (figure $2 \mathrm{a}$ ) can be removed by iodine-doping (figure $2 b$ ) so that the crystal is conductive all through the visible region of spectrum. The shorter rise and decay times in the iodine-doped crystals probably indicate that the drift mobility of charge carriers is greater in the doped crystals. Doping by growing the single crystal in the presence of the doping material is more stable and efficient than the doped crystals prepared by exposing the undoped crystals to the vapour of dopant. Thus it is shown that by suitably doping an organic semiconductor the photoconductivity could be very much enhanced.

\section{References}

Aoyagi Y et al 1971 J. Phys. Soc. Jap. 31164

Baughman R Het al 1977 J. Chem. Phys. 66401

Cox G A et al 1974 J. Phys. Chem. Solids 341655

Eley D D 1989 Mol. Cryst. Liq. Cryst. 1711

Fielding P E 1957 J. Chem. Phys. 26411

Gamo K et al 1968 J. Phys. Soc. Jap. 25431

Grigoryan L S et al 1984 Phys. Status Solidi (a) 84597

Gutmann F and Lyons L E 1981 Organic semiconductors (Malabar: Krieger)

Harikumar P S et al 1989 J. Mater. Sci. Lett. 8969

Heilmeier G H and Warfield G 1963 J. Chem. Phys. 38163

Hsu C and Labes M M 1974 J. Chem. Phys. 614640

Inokuchi H 1989 Mol. Cryst. Liq. Cryst. 17123

Liang C Y 1962 J. Chem. Phys. 37459

Lyons L E, Walsh J R and White J W 1960 J. Chem. Soc. 167

Lyons L E et al 1989 Mol. Cryst. Liq. Cryst. 17153

Minami N et al 1987 Jap. J. Appl. Phys. 261754

Moser F H and Thomas A L 1963 Phthalocyanine compounds (New York: Reinhold)

Orti E et al $1960 \mathrm{~J}$. Chem. Soc. 1960167

Orti E and Bredas J L 1988 J. Chem. Phys. 891009

Orti E, Bredas J L and Clarisse C 1990 J. Chem. Phys. 921228

Schoch K F et al $1988 \mathrm{~J}$. Vac. Sci. Technol. A6 155

Streetman B G 1980 Solid state electronic devices (Englewood Cliffs: Prentice Hall)

Waclawek W et al 1987 Thin Sold Films 1461

Wright J D 1989 Molecular crystals (New York: Cambridge)

Xavier F P et al 1995 Bull. Mater. Sci. 18269,283

Zabkowska-Waclawek M et al 1987 Mater. Sci. 13315 